NOTICE: This is the author's version of a work that was accepted for publication in Advances in Space Research. Changes resulting from the publishing process, such as peer review, editing, corrections, structural formatting, and other quality control mechanisms may not be reflected in this document. Changes may have been made to this work since it was submitted for publication. A definitive version was subsequently published in Advances in Space Research, Vol. 54, Issue 5. (2014). doi: 10.1016/j.asr.2013.03.007 


\title{
Ambiguity resolution performance with GPS and BeiDou for LEO formation flying
}

\author{
Sandra Verhagen ${ }^{1, *}$ \\ Department of Geoscience and Remote Sensing, Delft University of Technology \\ PO Box 5048, 2600 GA Delft, The Neterlands \\ Peter J.G. Teunissen* \\ Department of Geoscience and Remote Sensing, Delft University of Technology \\ PO Box 5048, 2600 GA Delft, The Neterlands \\ GNSS Research Centre, Curtin University, GPO Box U1987, Perth WA 6845, Australia
}

\begin{abstract}
The evolving BeiDou Navigation Satellite System constellation brings new opportunities for high-precision applications. In this contribution the focus will be on one such application, namely precise and instantaneous relative navigation of a formation of LEO satellites. The aim is to assess the ambiguity resolution performance with the future GPS and BeiDou constellations depending on system choice (GPS, BeiDou, or GPS+BeiDou), single- or dual-frequency observations, receiver noise, and uncertainties in ionosphere modelling. In addition, for the GPS+BeiDou constellation it will be shown how the growing BeiDou constellation in the years to come can already bring an important performance improvement compared to the GPS-only case. The performance will be assessed based on the percentage of time that the required precision can be obtained with a partial ambiguity resolution strategy.
\end{abstract}

Keywords: BeiDou; GPS; multi-GNSS; partial ambiguity resolution; LEO formation flying

\footnotetext{
*Corresponding author

Email address: a.a.verhagen@tudelft.nl (Sandra Verhagen)

${ }^{1}$ Tel. +31 (0)15 2784545
} 


\section{Introduction}

In the next decade users will have access to a truly multi-constellation Global Navigation Satellite System, with more than 100 satellites in orbit, divided over four individual constellations or systems. Those are the two currently available systems GPS and Glonass, and in addition the European Galileo and the Chinese BeiDou systems, both to be fully operational around 2020. The contribution of BeiDou to the relative navigation performance of Low Earth Orbiting (LEO) satellites will be the topic of this paper.

GPS has already been used for relative positioning of spacecraft in formation and for rendez-vous applications. The distance between the two spacecraft varied from very short (0-2km) for e.g. the ETS-7 (Kawano et al., 2001), PRISMA (D'Amico et al., 2006) and TanDEM-X (Montenbruck et al., 2008, 2011) missions, while for the GRACE mission (Tapley et al., 2005) the maximum distance was $220 \mathrm{~km}$. One of the goals of the PRISMA mission was to demonstrate real-time GPS-based autonomous formation flying capability, which was achieved to the level of $1 \mathrm{dm}$ (Gill et al., 2007). In all cases, an extended Kalman filter was applied with the disadvantage that dynamical models are required and the (re-)initialization time needs to be taken into account.

The aim here is to investigate whether (near) real-time availability of very precise relative position solution at $\mathrm{cm}$-level can be attained. This will be essential for rendez-vous missions and real-time monitoring systems, such as the TerraSAR-X and TanDEM-X formation (Moreira et al., 2004; Van Barneveld et al., 2009) and the Garada mission (Garada project, 2012; Li et al., 2011, 2012). The latter mission will be addressed in this contribution.

For very precise relative positioning in real-time, it is crucial that the carrier-phase ambiguities can be reliably fixed based on a single or few epochs of data. However, the high dynamical environment and the fact that all receivers are moving makes it more challenging to achieve this as compared to terrestrial applications, where usually a (network of) reference station(s) is available. Single-epoch positioning has the advantage that no dynamical models are required and losses-of-lock and/or cycle slips will not require reinitialization. Unfortunately, precise positioning with GPS-only will often not be possible due to low numbers of visible satellites, and because the noise characteristics of space-qualified receivers are often worse than those of high-quality geodetic receivers. Li et al. (2012) describe the development of the Namuru space receiver, which should overcome specific challenges re- 
lated to the LEO application and should enable cm-level real-time relative positioning.

The aforementioned challenges, however, may not be overcome with a GPS-only receiver. The added value of using the observations from more than one GNSS has been confirmed in many performance studies, see e.g. (Buist et al., 2010; Cao et al., 2008; Chen et al., 2009; O'Keefe et al., 2009; Rizos, 2009; Verhagen, 2007, 2010). Here, it will be specifically investigated how the relative positioning performance of a formation of LEOs may improve if BeiDou is used, either on its own or combined with GPS. Thereby different scenarios and circumstances will be considered. Firstly, the performance will be analysed for every 30 seconds of a full day and thus many different satellite geometries. Secondly, both the single- and dual-frequency case will be considered. Furthermore, different noise characteristics for both the observations and the residual ionosphere will be applied. Finally, it will be investigated how the growing BeiDou constellation may already contribute to improved performance.

It should be noted that instead of BeiDou, the European Galileo could be considered as well (Galileo, 2013). A similar performance improvement can be expected with combined GPS-Galileo, since the constellation of Medium Earth Orbiting satellites of BeiDou and Galileo are quite similar. And since in addition BeiDou will have the advantage of improved visibility over the Asia-Pacific region, Galileo has been left out the analysis in this contribution.

In the next section a brief overview of the GPS and BeiDou constellations will be given. In Section 3 the LEO formation flying mission to be considered is described. Section 4 presents the GNSS model and performance measures that will be used in Section 5 for the actual performance analyses.

\section{GPS and BeiDou}

GPS has been used for precise positioning applications for more than a decade. The constellation consists of at least 24 Medium Earth Orbiting (MEO) satellites (currently 31) divided over six orbital planes with an inclination of $55^{\circ}$ and at an altitude of $20,200 \mathrm{~km}$.

The BeiDou constellation is currently being deployed and is planned to reach full operational capability by 2020, (BeiDou, 2013; Yang et al., 2011). The full constellation should then consist of 5 geostationary (GEO), 3 Inclined Geo-Synchronous Orbiting (IGSO), and 27 MEO satellites. The orbital parameters of BeiDou are presented in Tables 1 and 2. 
Table 1: Orbital parameters BeiDou (full constellation), (Yang et al., 2011).

\begin{tabular}{llll}
\hline & GEO & IGSO & MEO \\
\hline \#satellites & 5 & 3 & $24+3$ spares \\
\#planes & 1 & 3 & 3 \\
semi-major axis [km] & 42,164 & 42,164 & 27,878 \\
inclination [deg] & 0 & 55 & 55 \\
right ascension [deg] & $58.75 ; 80 ; 110.5 ;$ & $118 ; 0 ; 238$ & $0 ; 120 ; 240$ \\
& $140 ; 160$ & & \\
mean anomaly [deg] & 0 & $0 ; 118 ;-120$ & see Table 2 \\
\hline
\end{tabular}

Table 2: Mean anomalies of BeiDou MEO satellites. *: spare satellite.

\begin{tabular}{ccc}
\hline Plane & $\begin{array}{c}\text { Right ascension } \\
{[\mathrm{deg}]}\end{array}$ & $\begin{array}{c}\text { Mean anomaly } \\
{[\mathrm{deg}]}\end{array}$ \\
\hline 1 & 0 & $0 ; 45 ; 90 ; 135 ; 180 ; 225 ; 270 ; 315 ; 10^{*}$ \\
2 & 120 & $15 ; 60 ; 105 ; 150 ; 195 ; 240 ; 285 ; 330 ; 55^{*}$ \\
3 & 240 & $30 ; 75 ; 120 ; 165 ; 210 ; 240 ; 300 ; 345 ; 105^{*}$ \\
\hline
\end{tabular}

Since the BeiDou constellation will not be complete immediately, it is interesting to see how the growing constellation can already start to contribute to enhanced positioning capabilities. This will be done by defining nine phases in the BeiDou deployment (these may not be corresponding with reality, since the exact launch schedule is unknown). In each phase, three MEO's are added to the constellation. Phase 0 corresponds to the GPS-only situation, while in phase 1 it is assumed that there are 3 GEO, 3 IGSO and 3 MEO satellites. In phase 2 and 3 the two remaining GEO satellites are added.

Table 3 gives an overview of the frequencies (to be) used by GPS and BeiDou. In this work, attention will be restricted to the open signals. Currently, dual-frequency dual-system space receivers are already being developed (Avanzi and Tortora, 2010; Li et al., 2012). Therefore single- and dualfrequency scenarios will be considered. For GPS, the L1 and L5 frequencies will be used.

First results with real BeiDou observations have been reported in e.g. Montenbruck et al. (2012); Nadarajah et al. (2012) and show that the BeiDou observations are of comparable accuracy as those of GPS and Galileo. In addition, enhanced ambiguity resolution capability with BeiDou was demon- 
Table 3: Overview of GPS and BeiDou frequencies. $\mathrm{O}=$ open signal, $\mathrm{X}=$ restricted access.

\begin{tabular}{l|l|cc}
\hline Band & Frequency & GPS & BeiDou \\
\hline L1 & $1575.42 \mathrm{MHz}$ & O & \\
B1 & $1561.098 \mathrm{MHz}$ & & O \\
B3 & $1268.52 \mathrm{MHz}$ & & X \\
L2 & $1227.60 \mathrm{MHz}$ & O & \\
B2 & $1207.14 \mathrm{MHz}$ & & O \\
L5 & $1176.45 \mathrm{MHz}$ & O & \\
\hline
\end{tabular}

\begin{tabular}{ll} 
Table 4: Orbit parameters of Garada satellites \\
\hline Semi major axis & $7,058.14 \mathrm{~km}$ \\
Inclination & $98.04^{\circ}$ \\
Right ascension & $-90.05^{\circ}$ \\
Eccentricity & $0^{\circ}$ \\
Mean anomaly & $0^{\circ}$ \\
Argument of perigee & $0^{\circ}$ \\
\hline
\end{tabular}

strated.

\section{LEO formation flying scenario and requirements}

In the frame of the Garada project (Garada project, 2012), a bistatic SAR mission is considered for a flood monitoring application. The mission comprises a constellation of 15-30 formations with 3 or 4 satellites each.

Figure 1 shows an example of one formation, with the red circle, $M$, representing the transmitting spacecraft and the two blue circles, $i$ and $j$, the receiving spacecraft, which are separated approximately $80 \mathrm{~km}$ from the transmitting satellite. The coherence between the observations made by the two receiving spacecraft is the parameter of interest, as it provides information on the presence of water and its motion between the two observation epochs. For the latter, it is required that the time separation between the observations by the two receiving satellites is in the order of $50 \mathrm{~ms}$. For the proposed Garada orbits, see Table 4, this implies a separation between the receiving platforms $i$ and $j$ of $0.5-1 \mathrm{~km}$. This baseline $i$-j needs to be determined very precisely, i.e. at centimeter level. 


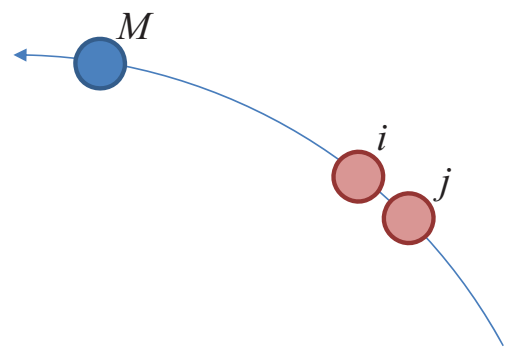

Figure 1: Garada formation with transmitting master satellite $(M)$ and two receiving satellites $(i$ and $j)$ with a separation of $0.5-1 \mathrm{~km}$.

\section{GNSS model and performance}

The linearized double-differenced (DD) observation equations for one epoch are given by

$$
\begin{aligned}
& p_{i j, f}^{l s}=-\left(u_{i}^{l s}\right)^{T} b+\mu_{f} I_{i j}^{l s}+e_{i j}^{l s} \\
& \phi_{i j, f}^{l s}=-\left(u_{i}^{l s}\right)^{T} b-\mu_{f} I_{i j}^{l s}+\lambda_{f} N_{i j, f}^{l s}+\epsilon_{i j}^{l s}
\end{aligned}
$$

The double-difference variates for receivers $i$ and $j$ and satellites $l$ and $s$ are defined as $x_{i j, f}^{l s}=\left(x_{j, f}^{s}-x_{j, f}^{l}\right)-\left(x_{i, f}^{s}-x_{i, f}^{l}\right)$. The code and carrier-phase observations are denoted $p$ and $\phi$, respectively. $u_{i}^{l s}$ is the differenced unit lineof-sight vector, $b$ the baseline vector between the receivers. $I$ is the ionosphere slant delay, $\lambda_{f}$ is the wavelength for frequency $f$, and $\mu_{f}=\left(\lambda_{f} / \lambda_{1}\right)^{2} . N$ the integer carrier-phase ambiguity and $e$ and $\epsilon$ are the remaining error terms for code and phase observations, respectively.

In Eq.(1) the GNSS orbit errors are assumed to be cancelled out, which is a realistic assumption for baselines up to tens of kilometers (Teunissen and Kleusberg, 1998, Chapter 5). Troposphere delays are absent at the orbital altitudes and are therefore not present in Eq.(1).

The ionosphere errors will cancel out in the double-differenced observations for short baselines of a few kilometers. However, for longer baselines the ionosphere delays must be taken into account. On the one hand, it will be beneficial or even required to use dual-frequency data then, since the ionosphere is a dispersive medium. Furthermore, the ionosphere weigthed model (Odijk, 2000) can be applied, which means that either corrections are applied 
or it is assumed that the differential delay is zero. With this approach the ionosphere parameters are eliminated from the observation equations, but the uncertainty of the corrections is taken into account.

The corrections can be based on a simplified ionospheric path delay model, which describes the differential path delay based on Lear (1987). This Lear mapping function has been specifically designed for spaceborne applications and takes into account that the ionospheric electron density maximum is at or below the orbital altitude of common LEO spacecraft. This implies that it suffices to estimate a single vertical delay $I_{z}$, which is mapped to the slant directions. The work on ionospheric path delay models for spaceborne GPS receivers flying in formation with long baselines is recently extended in Tancredi et al. (2011). Instead of estimating the vertical delays, it is also possible to use Vertical Total Electron Content (VTEC) maps from the International GNSS Service (Dow et al., 2005).

As mentioned, the accuracy of the ionosphere corrections must be taken into account. It is expressed as the between-receiver single-difference (SD) ionosphere standard deviation, which can thus be chosen zero or very small for short baselines, and will increase with baseline length. Van Barneveld et al. (2009) and Tancredi et al. (2011) reported accuracies of the ionosphere corrections of $3-4 \mathrm{~cm}$ for inter-satellite distances of $100-200 \mathrm{~km}$. For shorter distances, accuracies better than $1 \mathrm{~cm}$ were obtained.

The structure of the observation equations of the pseudorange and carrierphase observables is the same, only that the latter contains an ambiguity term. Since the phase standard deviation is a factor 100 smaller than the code standard deviation, this implies that the carrier-phase observations will start to act as very precise pseudorange observations if the ambiguities can be resolved. Therefore integer ambiguity resolution is the key to real-time and very precise relative positioning.

The DD GNSS model can be written as:

$$
y=A a+B b+e, \quad Q_{y y}
$$

where $y$ is the $m$-vector with DD code and phase observations, $b$ the 3 -vector with unknown baseline increments, $a$ the $n$-vector with ambiguities, and $e$ the $m$-vector with remaining error terms. $A$ and $B$ are the design matrices, and $Q_{y y}$ is the variance-covariance matrix of $y$.

If observations from more than one system are utilized, one needs to consider the fact that double-differencing with respect to one common reference 
satellite is only feasible if the same frequencies are employed and if the differential inter-system biases can be ignored Odijk (2013). In this contribution, the double-differences are formed with one reference satellite per system since the frequencies are not all identical.

Solving the GNSS model then comprises three steps. In the first step the integer nature of the carrier-phase ambiguities is discarded and the so-called float solution is obtained by e.g. an ordinary least-squares adjustment:

$$
\begin{aligned}
{\left[\begin{array}{l}
\hat{a} \\
\hat{b}
\end{array}\right] } & =\left[\begin{array}{ll}
Q_{\hat{a} \hat{a}} & Q_{\hat{a} \hat{b}} \\
Q_{\hat{b} \hat{a}} & Q_{\hat{b} \hat{b}}
\end{array}\right]\left[\begin{array}{l}
A^{T} \\
B^{T}
\end{array}\right] Q_{y y}^{-1} y \\
{\left[\begin{array}{ll}
Q_{\hat{a} \hat{a}} & Q_{\hat{a} \hat{b}} \\
Q_{\hat{b} \hat{a}} & Q_{\hat{b} \hat{b}}
\end{array}\right] } & =\left(\left[\begin{array}{l}
A^{T} \\
B^{T}
\end{array}\right] Q_{y y}^{-1}\left[\begin{array}{ll}
A & B
\end{array}\right]\right)^{-1}
\end{aligned}
$$

$\hat{a}$ and $\hat{b}$ are the float ambiguity and baseline estimators, respectively. $Q_{\hat{a} \hat{a}}$, $Q_{\hat{b} \hat{b}}$ and $Q_{\hat{b} \hat{a}}=Q_{\hat{a} \hat{b}}^{T}$ are the corresponding (co-)variance matrices.

In the second step the integer ambiguities are estimated and validated based on the ambiguity float solution. The fixed ambiguities are denoted $\check{a}$. After successful integer resolution, the ambiguity parameters can be assumed known, and as such an improved baseline solution, $\breve{b}$, can be obtained in the third step with:

$$
\check{b}=\hat{b}-Q_{\hat{b} \hat{a}} Q_{\hat{a} \hat{a}}^{-1}(\hat{a}-\check{a})
$$

Different integer estimators are available for the second step, but the integer least-squares (ILS) estimator is proven to be optimal, (Teunissen, 1999; Cai et al., 2009). The ILS solution is given by:

$$
\check{a}=\arg \min _{z \in \mathbb{Z}^{n}}\|\hat{a}-z\|_{Q_{\hat{a} \hat{a}}}^{2}
$$

An integer search is needed to determine $\check{a}$. The ILS procedure is efficiently mechanized in the LAMBDA method, see e.g. (Teunissen, 1993, 1995; Verhagen and Li, 2012). The efficiency is attained by the application of a decorrelating $Z$-transformation: $\hat{z}=Z^{T} \hat{a}$, with $Q_{\hat{z} \hat{z}}=Z^{T} Q_{\hat{a} \hat{a}} Z$. Recent contributions in the field of efficient ambiguity resolution are for instance (Chang et al., 2005; Jazaeri et al., 2012; Li et al., 2010; Wu et al., 2010).

It should be stressed that an incorrect integer ambiguity solution may cause large positioning errors, and therefore it is of crucial importance to validate the integer solution, which is generally done based on a discrimination test (Verhagen and Teunissen, 2006) and/or evaluation of the probability of 
correct integer estimation, the so-called success rate. For performance studies, the success rate is a very useful measure, as it can be computed without the need for actual observations. Exact evaluation, however, is not possible for ILS. Therefore in practice often the lower-bound based on the bootstrapping integer estimator is used. This estimator is known to perform close to optimal if applied to the decorrelated ambiguities $\hat{z}=Z^{T} \hat{a}$. Its success rate is equal to (Teunissen, 1998; Verhagen, 2005):

$$
P_{s, \mathrm{~B}}=\prod_{i=1}^{n}\left(2 \Phi\left(\frac{1}{2 \sigma_{\hat{z}_{i \mid I}}}\right)-1\right)
$$

with $\Phi(x)=\frac{1}{\sqrt{2 \pi}} \int_{-\infty}^{x} \exp \left\{-\frac{1}{2} t^{2}\right\} d t$ the cumulative normal distribution, and $\sigma_{\hat{z}_{i \mid I}}$ the standard deviation of the $i$ th least-squares ambiguity obtained through a conditioning on the previous $I=\{i+1, \ldots, n\}$ ambiguities. These $\sigma_{\hat{z}_{i \mid I}}$ are equal to the square root of the diagonal elements of diagonal matrix $D$ from the triangular factorization $Q_{\hat{z} \hat{z}}=L^{T} D L ; L$ is the corresponding unit lowertriangular matrix.

The success rate depends on the variance-covariance matrix of the float ambiguity solution $Q_{\hat{z} \hat{z}}$, which can be computed once the GNSS model is known as can be seen from Eq.(3). As such, the success rate depends on:

- receiver-satellite geometry (depends on time and location, as well as on which system is used)

- measurement noise (depends on GNSS signal and receiver quality)

- ionosphere uncertainty

- frequencies used (depends on system and receiver)

- number of observation epochs

since these factors drive the strength of the underlying GNSS model. In this study, the effect of the first four factors will be studied. The VISUAL software has been used for the design computations, (Verhagen, 2002).

In this work, the condition that the success rate should be larger than 99\% will be imposed in order to prevent that large positioning errors due to wrong fixing may occur. This implies that a precise fixed solution may often not be available instantaneously due to low precisions. It will be investigated whether a partial fixing scheme may be beneficial in terms of enhancing the 
availability. The idea is to fix only the largest possible subset of decorrelated ambiguities $\hat{z}$, such that the success rate requirement can indeed be met. As can be seen from Eq.(7), the bootstrapped success rate will namely decrease with increasing size of the subset since it is equal to a product of probabilities smaller than or equal to 1.

The uncertainty in the fixed integer ambiguity solution can be ignored due to the high success rate requirement. The variance-covariance matrix of $\check{b}$ is obtained by application of the propagation law of variances to Eq.(5):

$$
Q_{\check{b} \check{b}}=Q_{\hat{b} \hat{b}}-Q_{\hat{b}_{\hat{z}_{s}}} Q_{\hat{z}_{s} \hat{z}_{s}}^{-1} Q_{\hat{z}_{s} \hat{b}}
$$

where $\hat{z}_{s}$ is the fixed subset of decorrelated ambiguities.

The precision of relative positioning will here be evaluated with:

$$
\sigma_{b}=\sqrt{\operatorname{trace}\left(Q_{\check{b} \check{b}}\right)}
$$

\section{Results}

In this section the relative positioning performance will be assessed depending on system (GPS, BeiDou, GPS+BeiDou), number of frequencies (one or two), observation noise characteristics, and baseline length. The GPS and BeiDou constellations as described in Section 2 will be used. Garada satellite positions are generated for every 30 seconds of a full day.

By default an undifferenced code standard deviation of $30 \mathrm{~cm}$ in zenith direction is applied with exponential elevation-dependent weighting, see Figure 2. The undifferenced phase standard deviation is assumed to be a factor 100 smaller for all elevations. These values are realistic or even conservative if compared to values reported for the receivers on-board the GRACE, PRISMA and TANDEM-X/TERRASAR-X missions, cf. (Montenbruck and Kroes, 2003; Gill et al., 2007; Montenbruck et al., 2011), respectively. In the sequel, the code and phase noise is always specified as the standard deviation for an observation from the zenith direction. The between-receiver SD ionosphere standard deviation by default is equal to zero, since the Garada satellites will have a separation of at most $1 \mathrm{~km}$. In order to account for the high dynamical environment, the cut-off angle was set to $15^{\circ}$ so that the number of visible satellites will be somewhat lower as compared to most terrestrial applications. 


\section{Reference scenario}

Figures 3 and 4 show the performance for the single-frequency and dualfrequency systems (left and right, respectively). The availability is defined as the percentage of time that the baseline precision as defined in Eq.(9) is better than a certain value and with the success rate required to be larger than $99 \%$.

Figure 4 shows the number of fixed ambiguities versus the corresponding baseline precision for each epoch, i.e. it refers to the receiver-satellite geometry at the orbital position of the Garada satellites at that epoch. The color indicates the fraction of ambiguities, $F_{f i x}$, that could be fixed at that epoch:

$$
F_{f i x}=\frac{\# \text { fixed ambiguities }}{\# \text { float ambiguities }}
$$

$F_{\text {fix }}=1$ means that all ambiguities are fixed, $F_{\text {fix }}=0$ means that no ambiguities can be fixed and the baseline precision is equal to the float baseline precision. For each epoch the float baseline precision is shown in grey.

With single-frequency GPS, the baseline precision will be at the meter level. Figure 4 reveals that in general only a few ambiguities can be fixed, which is not enough to allow for a significant improvement of the corresponding fixed baseline precision as compared to the float baseline precision.

With single-frequency BeiDou the results are quite different. For 22\% of the epochs full ambiguity resolution is feasible, and for those epochs the fixed baseline precision is at the centimeter level. This is due to the improved satellite visibility in the Asia-Pacific region, which is defined such that China and Australia are completely covered, see Figure 5. Figure 6 shows the baseline precision as function of orbital position (longitude and latitude), which clearly shows that indeed higher precisions are obtained in the aforementioned region. It may even suffice to use BeiDou-only if this is the region of interest.

With observations on two frequencies the availability will be largely improved for both GPS-only and BeiDou-only. A precision of a few centimeters can be available instantaneously for about $95 \%$ of the time.

With a combined GPS+BeiDou constellation, the performance can be further improved due to enhanced satellite visibility, allowing for centimeter level relative navigation for about $97 \%$ of the time with single-frequency observations, and 100\% for the dual-frequency case (see Figure 3).

Figure 4 allows to analyse the contribution of partial ambiguity resolution. It follows that only if more than $50 \%$ of the ambiguities can be fixed (cyan 


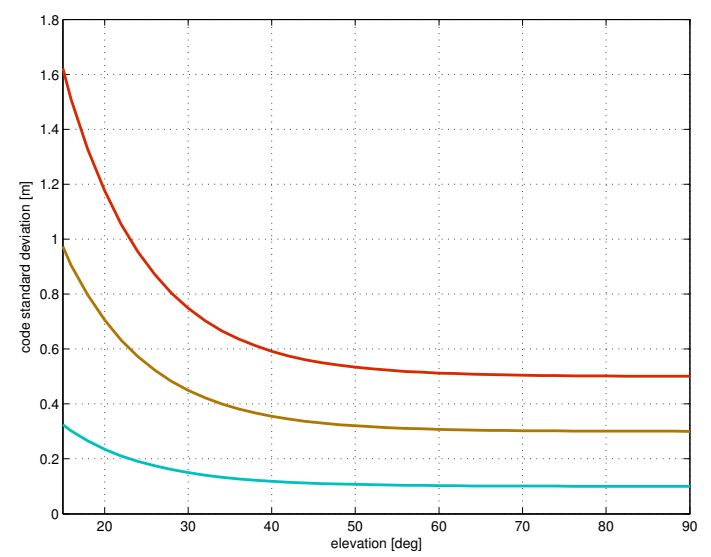

Figure 2: Code standard deviation [m] as function of elevation for three values of the standard deviation in zenith direction, namely 10,30 and $50 \mathrm{~cm}$.

dots), this will lead to improved baseline precision. However, full ambiguity resolution is required for centimeter level accuracies with single epoch observations.

\section{Contribution of growing BeiDou constellation}

The contribution of BeiDou in its nine different phases as defined in Section 2 is shown in Figure 7. Only the combined GPS+BeiDou constellation is considered here. The baseline precision is required to be better than $15 \mathrm{~mm}$. For the single-frequency scenario this contribution becomes significant already at an early stage. The availability increases from $0 \%$ with GPS-only to more than $50 \%$ with half of the BeiDou constellation. For the dual-frequency case the availability is already close to $99 \%$ with half of the BeiDou constellation, compared to $90 \%$ with GPS-only. Obviously, it will be interesting to start using combined GPS+BeiDou already now even though the constellation is far from complete.

\section{Impact of noise}

The ambiguity success rate largely depends on the observation noise. Figure 8 shows how this propagates into the availability for both the single- and dual-frequency cases with a required baseline precision of $15 \mathrm{~mm}$. The availability is shown as function of the code noise; the carrier-phase noise again is always a factor 100 smaller. 

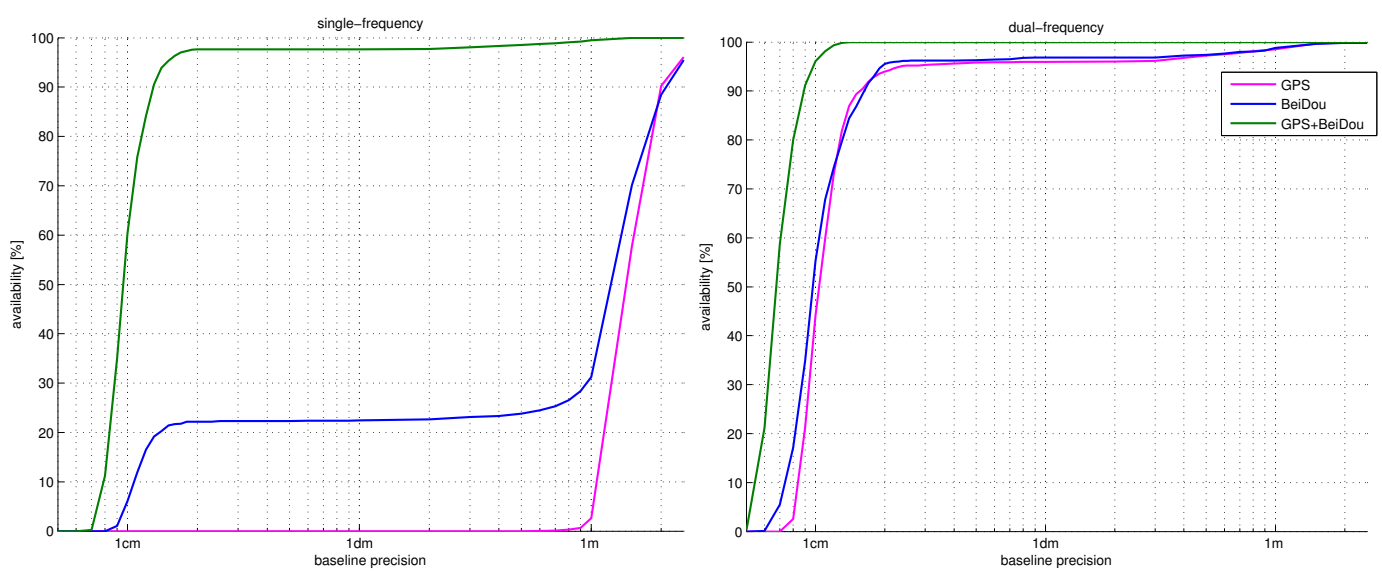

Figure 3: Availability as function of required baseline precision (standard deviation of fixed baseline) for single-epoch GPS, BeiDou, and GPS+BeiDou. Code standard deviation is $30 \mathrm{~cm}$, success rate is required to be $>99 \%$. Left: single-frequency; Right: dual-frequency.

With combined GPS+BeiDou instantaneous positioning is possible as long as the code noise is below $25 \mathrm{~cm}$ and $35 \mathrm{~cm}$ for the single- and dual-frequency case, respectively. Single-epoch precise positioning is not feasible with singlefrequency GPS-only or BeiDou-only for $20 \%$ of the time even with very high precision measurements. With two frequencies the situation is much improved. If the code noise is below $25 \mathrm{~cm}$, the availability will be above $90 \%$. Interestingly, instantaneous positioning is possible with low-grade receivers, i.e. high noise characteristics, with a dual-frequency GPS+BeiDou constellation.

The dashed lines in Figure 8 show the availability for the Asia-Pacific region only. Due to the additional IGSO and GEO satellites in this region the availability can be very high with BeiDou-only, especially with the dualfrequency scenario.

\section{Impact of baseline length}

A longer baseline will imply more uncertainty in the ionosphere corrections/assumptions. Figure 9 shows how the availability decreases as this uncertainty becomes larger. Only the dual-frequency scenario is considered since for longer baselines it cannot be expected to obtain good results with single-frequency receivers. The baseline precision requirement is chosen less stringent, namely $15 \mathrm{~cm}$, as it is expected that for the longer baselines decimeter-level precision is sufficient since the relative positions are not 

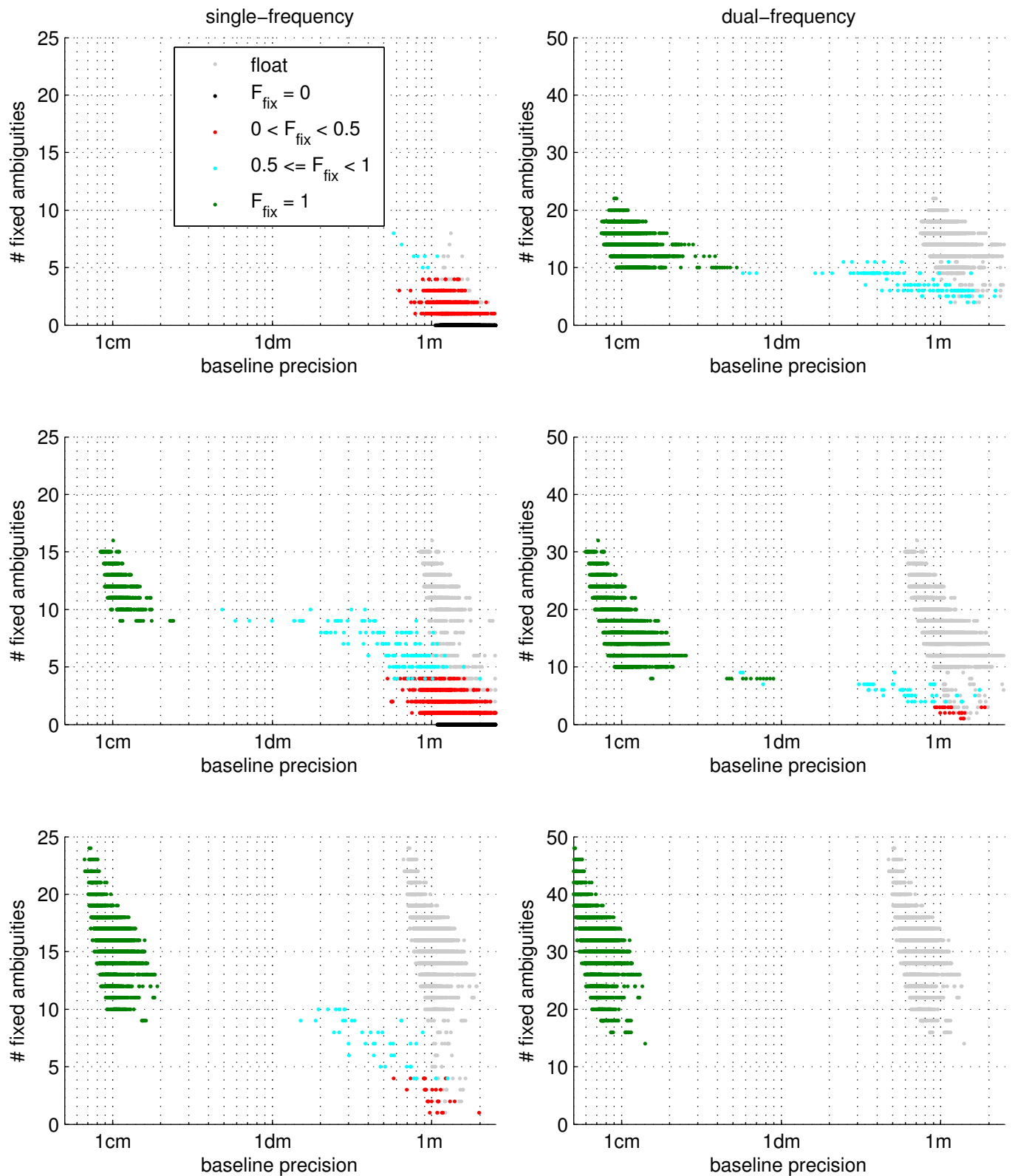

Figure 4: Number of fixed ambiguities versus baseline precision (standard deviation of fixed baseline) for single-epoch GPS (top), BeiDou (centre), and GPS+BeiDou (bottom), success rate is required to be $>99 \%$. The colors indicate the fraction of fixed ambiguities, $F_{f i x}$. The grey dots show the corresponding float baseline precisions. Left: single-frequency; Right: dual-frequency. 


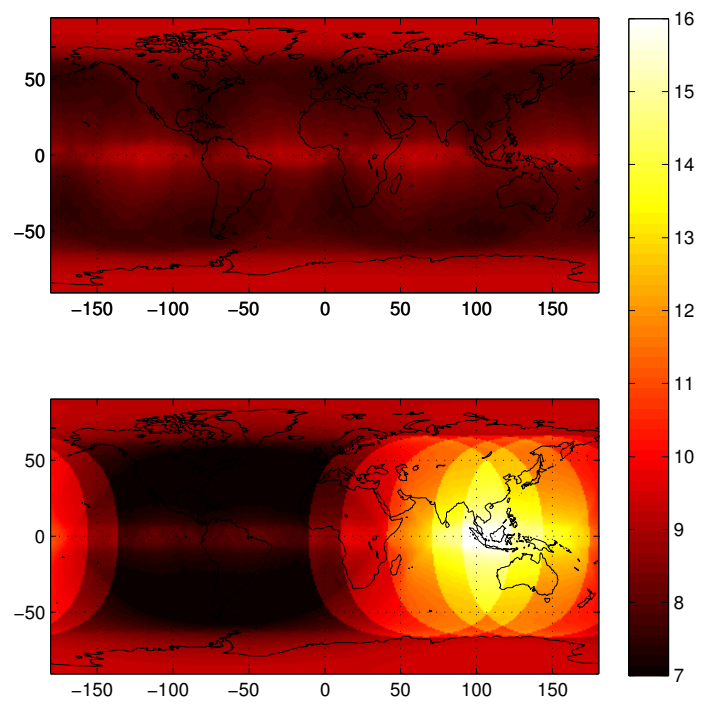

Figure 5: Average number of visible satellites over one day for GPS (top) and BeiDou (bottom).

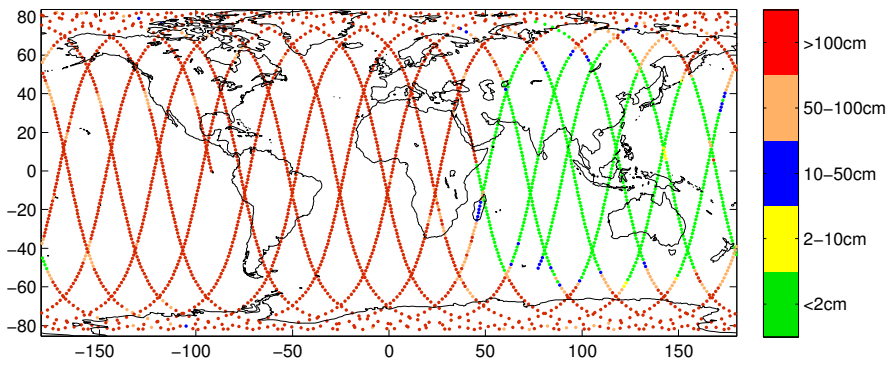

Figure 6: Baseline precision (standard deviation of fixed baseline) with single-epoch, single-frequency BeiDou, success rate is required to be $>99 \%$. 

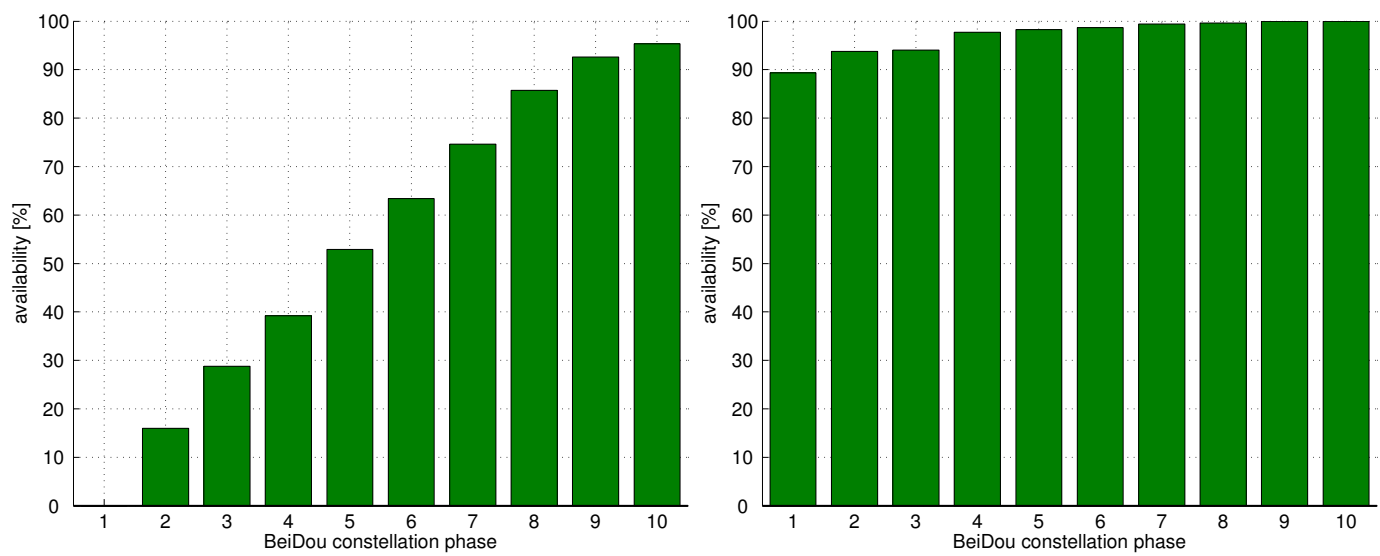

Figure 7: Availability as function of the growing BeiDou constellation (phase 0 is GPSonly, phase 9 is full operational capability) for single-epoch GPS+BeiDou. Required baseline precision is $15 \mathrm{~mm}$, success rate is required to be $>99 \%$. Left: single-frequency; Right: dual-frequency.
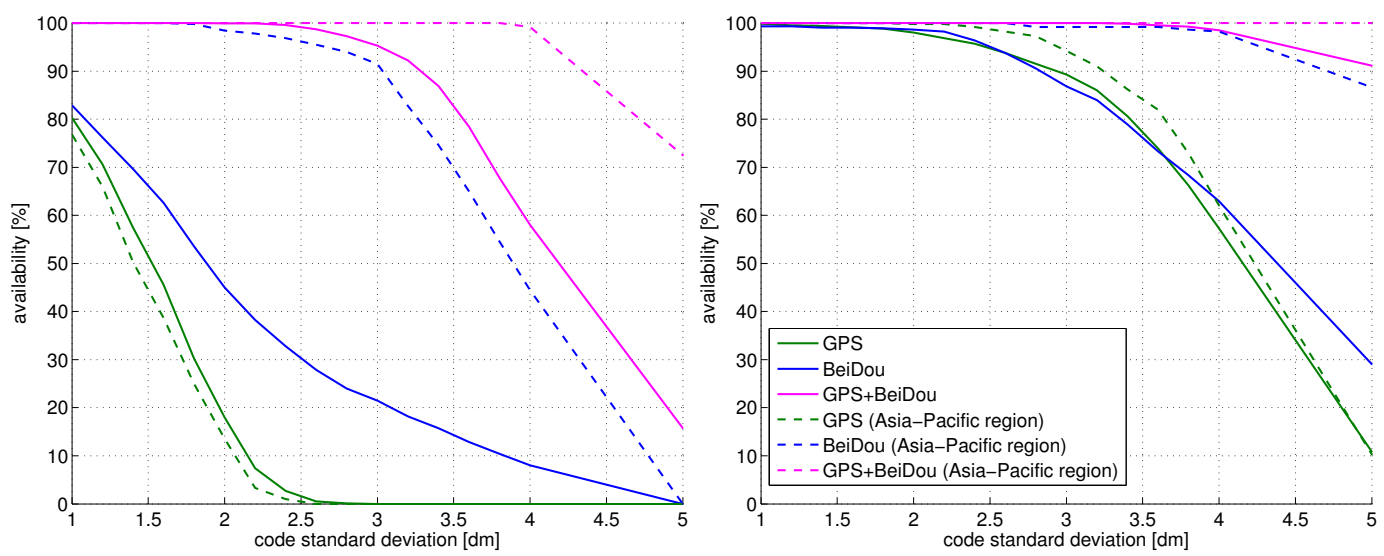

Figure 8: Availability as function of the code standard deviation [dm] for single-epoch GPS, BeiDou, and GPS+BeiDou. Required baseline precision is $15 \mathrm{~mm}$, success rate is required to be $>99 \%$. Solid lines: global availability. Dashed lines: availability in AsiaPacific region. Left: single-frequency; Right: dual-frequency. 


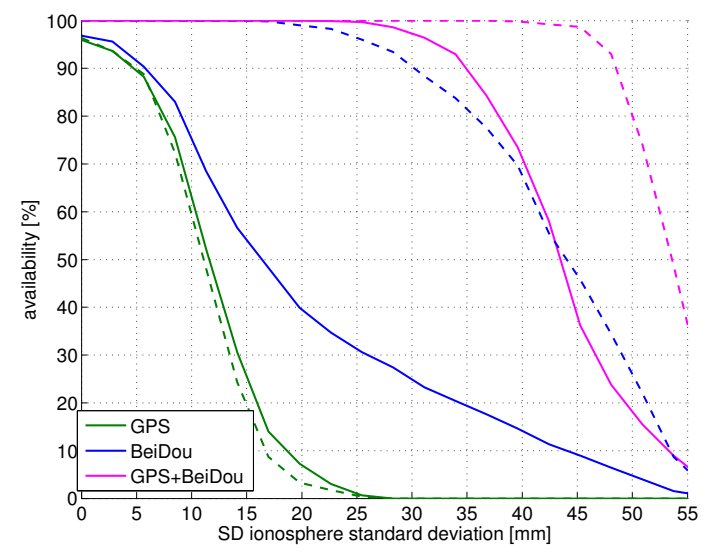

Figure 9: Availability as function of the SD ionosphere standard deviation [mm] for singleepoch, dual-frequency GPS, BeiDou, and GPS+BeiDou. Required baseline precision is $15 \mathrm{~cm}$, success rate is required to be $>99 \%$. Solid lines: global availability. Dashed lines: availability in Asia-Pacific region.

needed for the coherence measurements.

Instantaneous positioning with combined GPS+BeiDou will still be feasible with an SD ionosphere standard deviation of $25 \mathrm{~mm}$, which will be a realistic value for baselines of tens of kilometers if compared to the accuracies found in (Van Barneveld et al., 2009; Tancredi et al. , 2011). However, single-epoch precise positioning with a single system will only be possible for very short baselines, since the availability drops rapidly with increasing ionosphere uncertainty. On the other hand, if only the Asia-Pacific region is to be covered, BeiDou-only will allow for instantaneous ambiguity resolution as long as the ionosphere uncertainty is less than $20 \mathrm{~mm}$. With a combined GPS+BeiDou constellation the required performance may be obtained for even longer inter-satellite distances.

For longer baselines it has also been studied how many epochs would be required in order to meet the baseline precision requirement for at least $99 \%$ of the time. This is shown in Figure 10 for different ionosphere uncertainties and a precision requirement of $15 \mathrm{~cm}$ and $5 \mathrm{~cm}$, respectively. Increasing uncertainty in the ionosphere corrections can be interpreted as corresponding to increasing baseline length. For the less stringent precision requirement, it can be seen that with combined GPS+BeiDou only one or two epochs suffice, even for longer baselines. With a single GNSS it is obvious that more epochs are needed with increasing baseline length, although for short baselines also 

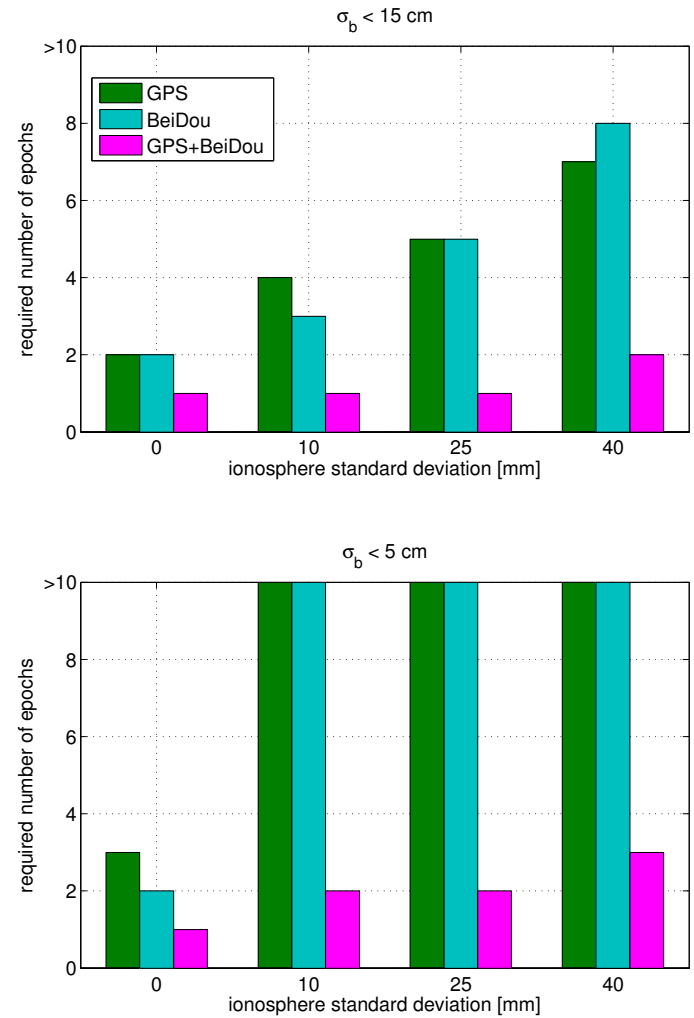

Figure 10: Required number of epochs as function of the SD ionosphere standard deviation [mm] for dual-frequency GPS, BeiDou, and GPS+BeiDou. Requirements: $99 \%$ availability with a baseline precision better than $15 \mathrm{~cm}$ (top) and $5 \mathrm{~cm}$ (bottom), and success rate $>99 \%$. 
then only a few epochs may suffice. Once the precision requirement is set to $5 \mathrm{~cm}$, it becomes clear that only combined GPS+BeiDou will allow to reach the performance rapidly.

In conclusion, it can be stated that high precision relative positioning with GNSS will be feasible in (near) real-time. For longer inter-satellite distances it will be necessary to use dual-frequency observations from two systems, while for short distances even one system may suffice depending on the actual requirements as well as on the receiver noise and uncertainty in the ionosphere corrections.

\section{Concluding remarks}

The performance study presented in this contribution clearly shows the added value of BeiDou as it comes to real-time precise relative positioning of LEO satellites. A dramatic improvement in availability will be possible with a combined GPS+BeiDou constellation, already in the deployment phase of BeiDou. If the area of interest is restricted to the Asia-Pacific region even BeiDou-only may suffice for shorter baselines once the constellation is complete.

The results in this contribution show the theoretical performance as depending on the choice and availability of GNSS, the number of frequencies, the observation noise and the uncertainty in the ionosphere corrections. Once realistic values for noise and accuracy of the ionosphere corrections are available for future missions, this will allow to assess the expected relative navigation performance.

Especially for space applications the low number of visible satellites, high observation noise and multipath may hinder successful single-epoch ambiguity resolution. The problem of bad satellite visibility can be overcome by using a dual-constellation, as is demonstrated here. Different figures for the measurement noise have been applied to study the sensitivity of the ambiguity resolution performance. This revealed that high noise will have a big impact with a single GNSS, but less so if GPS+BeiDou observations are available. The problem of multipath-induced errors has been left out of this study, since these errors depend very much on the platform and satellite geometry. All in all, it can be concluded that from a theoretical point of view very precise relative positioning with single-epoch GNSS will be feasible with a dual-constellation GNSS. 
In this contribution only formation flying with a single antenna configuration on each LEO platform is considered. The results can be significantly enhanced with two or more antennas with a known geometry on each platform. Multi-antenna configurations allow for GNSS carrier-phase based attitude determination. It has been shown that ambiguity resolution success rates will be dramatically improved by exploiting the known antenna geometry as mechanized in the Multivariate-Constrained LAMBDA method, (Buist et al., 2010).

\section{Acknowledgments}

This work has been executed in the framework of two projects. Firstly, the Positioning Program Project 1.01 "New carrier phase processing strategies for achieving precise and reliable multi-satellite, multi-frequency GNSS/RNSS positioning in Australia" of the Cooperative Research Centre for Spatial Information. Secondly, the "Garada" project on SAR formation flying, supported by the Australian Space Research Program.

Peter J.G. Teunissen is the recipient of an Australian Research Council (ARC) Federation Fellowship (project number FF0883188). All this support is gratefully acknowledged.

\section{References}

Avanzi A, \& Tortora P, Design and implementation of a new spaceborne FPGA-based dual frequency GPS and Galileo software defined receiver. Proceedings of 5th ESA Workshop on Satellite Navigation Technologies, NAVITEC 2010, 2010.

BeiDou, http://en.beidou.gov.cn/index.html, visited January 2013.

Buist P, Teunissen PJG, Giorgi G, \& Verhagen S, Multivariate Bootstrapped Relative Positioning of Spacecraft Using GPS L1/Galileo E1 Signals. Advances in Space Research, 47(5): 770-785, 2010.

Cai J, Grafarend E, \& Hu C, The total optimal criterion in solving the mixed integer linear model with GNSS carrier phase observations,. GPS Solutions, 13: 221-230, 2009. 
Cao W, O'Keefe K, \& Cannon ME, Evaluation of Compass Ambiguity Resolution Performance Using Geometric-Based Techniques with Comparison to GPS and Galileo. Proceedings of ION GNSS 2008, Savannah GA, pp.1688-1697, 2008.

Chang X, Yang X, \& Zhou T, MLAMBDA: a modified LAMBDA method for integer least-squares estimation. Journal of Geodesy, 79: 552-565, 2005.

Chen HC, Huang YS, Chiang KW, Ming Y, \& Ruey-Juin R, The performance comparison between GPS and Beidou-2/Compass: a perspective from Asia. J. Chin. Inst. Eng., 32(5): 679-689, 2009.

D'Amico S, Gill E, Garcia O, \& Montenbruck O, GPS-based real-time navigation for the PRISMA formation flying mission. Proceedings of 3rd ESA Workshop on Satellite Navigation Technologies, NAVITEC 2006, 2006.

Dow JM, Neilan RE, \& Gendt G, The International GPS Service (IGS): celebrating the 10th anniversary and looking to the next decade. Advances in Space Research, 36(3): 320-326, 2005.

Galileo, http://ec.europa.eu/enterprise/policies/satnav/galileo/index_en.htm, visited January 2013.

Garada project, http://www.garada.unsw.edu.au

Gill E, D'Amico S, \& Montenbruck O, Autonomous Formation Flying for the PRISMA Mission. Journal of Spacecraft and Rocket, 44(3): 671-681, 2007.

Jazaeri S, Amiri-Simkooei AR, \& Sharifi MA, Fast integer least-squares estimation for GNSS high-dimensional ambiguity resolution using lattice theory. Journal of Geodesy, 86(2): 123-136, 2012.

Kawano I, Mokuno M, Kasai T, \& Suzuki T, First autonomous rendezvous using relative GPS navigation by ETS-VII. Navigation, 48(1): 49-56, 2001.

Lear WM, GPS Navigation for Low-Earth Orbiting Vehicles. NASA 87-FM2, Rev. 1; JSC-32031, Lyndon B. Johnson Space Center; Houston, Texas, 1987.

Li B., Shen Y, \& Feng Y, Fast GNSS ambiguity resolution as an ill-posed problem. Journal of Geodesy, 84: 683-698, 2010. 
Li R, Jiao Y, Li Y, \& Rizos C, Simulation platform for relative navigation using GPS carrier phase measurements for satellite formation flying missions. Proceedings of IGNSS Symposium, paper 41, Sydney, Australia, 15-17 November, 2011.

Li Y, Glennon EP, Li R, Jiao Y, \& Dempster AG, Development of a spaceborne GPS receiver for precise relative navigation of formation flying small satellites. Lecture Notes in Electrical Engineering, Vol. 161, 467-476, 2012.

Montenbruck O, \& Kroes R, In-flight performance analysis of the CHAMP BlackJack GPS Receiver. GPS Solutions, 7(2): 74-86, 2003.

Montenbruck O, Kahle R, D'Amico S, \& Ardaens JS, Navigation and control of the TanDEM-X formation. Journal of Astronautical Sciences, 56(3): 341-357, 2008.

Montenbruck O, Wermuth M, \& Kahle R, GPS Based Relative Navigation for the TanDEM-X Mission - First Flight Results. Navigation, 58(4): 293-304, 2011.

Montenbruck O, Hauschild A, Steigenberger P, Hugentobler U, Teunissen PJG, \& Nakamura S, Initial assessment of the COMPASS/BeiDou-2 regional navigation satellite system. GPS Solutions, DOI: 10.1007/s10291012-0272-x, 2012.

Moreira A, Krieger G, Hajnsek I, Hounam D, Werner M, Riegger S, \& Settelmeyer E, TanDEM-X: a TerraSAR-X add-on satellite for singlepass SAR interferometry. Proceedings of IGARSS'04, IEEE International, Vol.2, pp. 1000-1003, 2004.

Nadarajah N, Teunissen PJG, Buist P, \& Steigenberger P, First results of instantaneous GPS/Galileo/COMPASS attitude determination. Proceedings of 6th ESA Workshop on Satellite Navigation Technologies, NAVITEC 2012, 2012.

Odijk D, Weighting ionospheric corrections to improve fast GPS positioning over medium distances. Proceedings of ION GPS 2000, Salt Lake City UT, pp.1113-1123, 2000. 
Odijk D, \& Teunissen PJG, Characterization of between-receiver GPSGalileo inter-system biases and their effect on mixed ambiguity resolution. GPS Solutions, DOI: 10.1007/s10291-012-0298-0, 2012.

O’Keefe K, Petovello MG, Cao W, Lachapelle G, \& Guyader E, Comparing Multi-carrier Ambiguity Resolution Methods for Geometry-Based GPS and Galileo Relative Positioning and their Application to Low Earth Orbiting Satellite Attitude Determination. International Journal of Navigation and Observation, Vol. 2009, Article ID 592073, 15 pages, 2009.

Rizos C, Multi-constellation GNSS/RNSS from the perspective of high accuracy users in Australia. Journal of Spatial Science, 53(2): 29-63, 2009.

Tancredi U, Renga A, \& Grassi M, Ionospheric path delay models for spaceborne GPS receivers flying in formation with large baselines. Advances in Space Research, 48(3): 507-520, 2011.

Tapley B, Ries J, Bettadpur S, Chambers D, Cheng M, Condi F, Gunter B, Kang Z, Nagel P, Pastor R, Pekker T, Poole S, \& Wang F, GGM02 An improved Earth gravity field model from GRACE. Journal of Geodesy, 70(1-2): 467-478, 2005.

Teunissen PJG, Least squares estimation of the integer GPS ambiguities. Invited lecture, Section IV Theory and Methodology, IAG General Meeting, Beijing, 1993.

Teunissen PJG, The least-squares ambiguity decorrelation adjustment: a method for fast GPS integer ambiguity estimation. Journal of Geodesy, 70: 65-82, 1995.

Teunissen PJG, Success probability of integer GPS ambiguity rounding and bootstrapping. Journal of Geodesy, 72, 606-612, 1998.

Teunissen PJG, An optimality property of the integer least-squares estimator. Journal of Geodesy, 73(11): 587-593, 1999.

Teunissen PJG, \& Kleusberg A, GPS for Geodesy. 2nd Edition, Springer, 1998.

Van Barneveld PWL, Montenbruck O, \& Visser PNAM, Epochwise prediction of GPS single differenced ionospheric delays of formation flying spacecraft. Advances in Space Research, 44(9): 987-1001, 2009. 
Verhagen S, Studying the performance of Global Navigation Satellite Systems: A new software tool. GPS World, 13: 60-65, 2002.

Verhagen S, On the reliability of integer ambiguity resolution. Navigation, 52(2): 99-110, 2005.

Verhagen S, Reliable positioning with the next generation Global Navigation Satellite Systems. Proceedings of 3rd International Conference on Recent Advances in Space Technologies, RAST '07, pp. 618-623, 2007.

Verhagen S, Odijk D, Teunissen P, \& Huisman L, Performance improvement with low-cost multi-GNSS receivers. Proceedings of 5th ESA Workshop on Satellite Navigation Technologies, NAVITEC 2010, 2010.

Verhagen S, \& Li B, LAMBDA software package: Matlab implementation, Version 3.0. Available from http://gnss.curtin.edu.au, 2012.

Verhagen S \& Teunissen PJG, New global navigation satellite system ambiguity resolution method compared to existing approaches. Journal of Guidance, Control, and Dynamics, 29: 981-991, 2006.

Wu Y, Jin SG, Wang Z, \& Liu J, Cycle slip detection using multi-frequency GPS carrier phase observations: A simulation study. Advances in Space Research, 46(2): 144-149, 2010.

Yang YX, Li JL, Xu JY, Tang J, Gue HR, \& He HB, Contribution of the Compass satellite navigation system to global PNT users. Chinese Science Bulletin, 56: 2813-2819, 2011. 